\title{
Explicit approximations to estimate the perturbative diffusivity in the presence of convectivity and damping (Part 2): Semi-infinite cylindrical approximations
}

\author{
M. van Berkel ${ }^{1,2,3,4}$, G.M.D. Hogeweij ${ }^{3}$, N. Tamura ${ }^{1}$, \\ H.J. Zwart ${ }^{5,6}$, S. Inagaki ${ }^{7}$, M.R. de Baar $^{3,4}$, and K. Ida ${ }^{1}$ \\ ${ }^{1}$ National Institute for Fusion Science, \\ 322 Oroshi-cho, Toki-city, Gifu, 509-5292, Japan \\ ${ }^{2}$ Fellow of the Japan Society for the Promotion of Science (JSPS) \\ ${ }^{3}$ FOM Institute DIFFER-Dutch Institute for Fundamental Energy Research, \\ Association EURATOM-FOM, Trilateral Euregio Cluster, \\ PO Box 1207, 3430 BE Nieuwegein, The Netherlands \\ ${ }^{4}$ Eindhoven University of Technology, Dept. of Mechanical Engineering, \\ Control Systems Technology group, PO Box 513, \\ 5600 MB Eindhoven, The Netherlands \\ ${ }^{5}$ Eindhoven University of Technology, \\ Dept. of Mechanical Engineering, Dynamics and Control group, \\ PO Box 513, 5600 MB Eindhoven, The Netherlands \\ ${ }^{6}$ Department of Applied Mathematics, \\ University of Twente, P.O. Box 217, \\ 7500 AE Enschede, The Netherlands and \\ ${ }^{7}$ Research Institute for Applied Mechanics, \\ Kyushu University, Kasuga 816-8580, Japan
}




\begin{abstract}
In this paper, a number of new explicit approximations are introduced to estimate the perturbative diffusivity $(\chi)$, convectivity $(V)$, and damping $(\tau)$ in a cylindrical geometry. For this purpose the harmonic components of heat waves induced by localized deposition of modulated power are used. The approximations are based upon the heat equation in a semi-infinite cylindrical domain. The approximations are based upon continued fractions, asymptotic expansions, and multiple harmonics.

The relative error for the different derived approximations is presented for different values of frequency, transport coefficients, and dimensionless radius. Moreover, it is shown how combinations of different explicit formulas can yield good approximations over a wide parameter space for different cases, such as no convection and damping, only damping, and both convection and damping.

This paper is the second part (Part 2) of a series of three papers. In Part 1 the semi-infinite slab approximations have been treated. In Part 3 cylindrical approximations are treated for heat waves traveling towards the center of the plasma.
\end{abstract}




\section{INTRODUCTION}

This paper, Part 2 of a series of three papers, deals with semi-infinite cylindrical approximations that can be used to calculate the perturbative thermal diffusion coefficient, convectivity, and damping from the experimental data of heat pulse propagation. For a general introduction of the series of three papers the reader is referred to [1].

This paper is structured as follows. Section II gives an overview of the relevant assumptions and models used for perturbative transport analysis. In Section III, three methods are introduced to find approximations for $\chi, V$, and $\tau$, i.e., continued fractions, asymptotic expansions, and multiple harmonics. Section [V] gives an overview of possible explicit approximations under various assumptions. The different approximations are compared for different values of the transport coefficients, dimensionless radius, and frequency. In practice, the transport coefficients are unknown. Therefore, in Section $\mathrm{V}$ a method is introduced to select and test the accuracy of the approximation without using knowledge of the actual transport coefficients. Finally, in Section VI the main results are summarized and discussed.

\section{MODELING OF THERMAL TRANSPORT}

In this section, the main assumptions and models used for perturbative transport analysis of the electron transport are summarized.

\section{A. Perturbative transport analysis}

Linearized thermal transport inside a fusion reactor is often modeled as radial (1D) transport in a cylinder due to the magnetic confined plasma topology [1-3]

$$
\begin{aligned}
\frac{3}{2} \frac{\partial}{\partial t}(n T)= & \frac{1}{\rho} \frac{\partial}{\partial \rho}\left(n \rho \chi(\rho) \frac{\partial T}{\partial \rho}+n \rho V(\rho) T\right) \\
& -\frac{3}{2} n \tau_{\text {inv }}(\rho) T+P_{\text {mod }}
\end{aligned}
$$

where $\chi$ is the diffusivity, $V$ the convectivity, $\tau_{i n v}$ the (inverse) damping $\left(\tau_{i n v}=1 / \tau\right), T$ denotes the electron temperature, $n$ the density, $\rho$ the dimensionless radius, and $P_{\text {mod }}$ a perturbative heat source. However, a number of assumptions are necessary to derive direct expressions for $\chi$, which are standard in the literature [2, 4]. These assumptions simplify 
(1) such that analytic solutions can be derived for (1). Only measurements are considered for which the transients due to the initial condition can be neglected. It is assumed that the parameters are constant with respect to time and $\rho$, which is also referred to as being homogenous or uniform [4]. Finally, only spatial regions are considered where $P_{\text {mod }}=0$, i.e., outside the region where the heating is deposited to perturb the plasma. Here, it is additionally assumed that the density $n$ is constant with respect to $\rho$. Under these assumptions (1) can be transformed into the Laplace domain yielding

$$
\begin{gathered}
\frac{3}{2}\left(s+\tau_{\text {inv }}\right) \Theta(\rho, s)= \\
\frac{1}{\rho} \frac{d}{d \rho}\left(\rho \chi \frac{\partial \Theta(\rho, s)}{\partial \rho}+\rho V \Theta(\rho, s)\right),
\end{gathered}
$$

where $\Theta$ is the Laplace transformed temperature $T$ and $s$ is the Laplace variable. This complex valued Ordinary Differential Equation (ODE) can be solved analytically [5, 6]:

$$
\begin{gathered}
\Theta(\rho, s)=e^{\lambda_{1} \rho} D_{1}(s) \Psi\left(\frac{\lambda_{2}}{\lambda_{2}-\lambda_{1}}, 1,\left(\lambda_{2}-\lambda_{1}\right) \rho\right) \\
+e^{\lambda_{1} \rho} D_{2}(s) \Phi\left(\frac{\lambda_{2}}{\lambda_{2}-\lambda_{1}}, 1,\left(\lambda_{2}-\lambda_{1}\right) \rho\right)
\end{gathered}
$$

where

$$
\lambda_{1,2}=-\frac{V}{2 \chi} \mp \sqrt{\left(\frac{V}{2 \chi}\right)^{2}+\frac{3}{2} \frac{s+\tau_{i n v}}{\chi}} .
$$

The functions $\Phi(\rho, s)$ and $\Psi(\rho, s)$ denote the Confluent Hypergeometric Functions of the first and the second kind, respectively. These functions are also often denoted as ${ }_{1} F_{1}$ and $U$, and are extensively described in [7-9]. The boundary constants are denoted by $D_{1}(s)$ and $D_{2}(s)$. This solution in terms of Confluent Hypergeometric Functions may not be so familiar, but if simplified by assuming $V=0$, the well-known solutions in terms of the modified Bessel functions of the first kind $I_{\nu}$ and the second kind $K_{\nu}$ of order $\nu=0$ are found. In particular, if $V=0$, then $\lambda_{1}=-\lambda_{2}, \lambda_{2} /\left(\lambda_{2}-\lambda_{1}\right)=1 / 2$, and $\Phi(\rho, s)$ and $\Psi(\rho, s)$ result in [7]

$$
\begin{gathered}
\Phi\left(\frac{1}{2}, 1,\left(\lambda_{2}-\lambda_{1}\right) \rho\right)= \\
\exp \left(\frac{1}{2}\left(\lambda_{2}-\lambda_{1}\right) \rho\right) I_{0}\left(\frac{1}{2}\left(\lambda_{2}-\lambda_{1}\right) \rho\right)
\end{gathered}
$$

and

$$
\Psi\left(\frac{1}{2}, 1,\left(\lambda_{2}-\lambda_{1}\right) \rho\right)=
$$




$$
\exp \left(\frac{1}{2}\left(\lambda_{2}-\lambda_{1}\right) \rho\right) \frac{K_{0}\left(\frac{1}{2}\left(\lambda_{2}-\lambda_{1}\right) \rho\right)}{\sqrt{\pi}}
$$

So (3) simplifies to

$$
\begin{gathered}
\Theta(\rho, s)=\frac{D_{1}(s)}{\sqrt{\pi}} K_{0}\left(\frac{1}{2}\left(\lambda_{2}-\lambda_{1}\right) \rho\right) \\
+D_{2}(s) I_{0}\left(\frac{1}{2}\left(\lambda_{2}-\lambda_{1}\right) \rho\right) .
\end{gathered}
$$

This solution is well known and is studied and referenced in e.g. [6, 10].

These solutions are used to derive hands-on models, which can be used to derive explicit approximations of $\chi$.

\section{B. Logarithmic temperature derivative and transfer function}

An explicit solution is difficult to derive on the basis of the two eigenfunctions $\Phi(\rho, s)$ and $\Psi(\rho, s)$. Therefore, an additional simplification step is necessary to be able to eliminate one eigenfunction. One eigenfunction is eliminated by assuming a semi-infinite domain, which is defined as follows, if $\rho \rightarrow \infty$, then $\Theta \rightarrow 0$. This means that at $\rho=\infty$ all perturbations need to have vanished.

In case $V=0$ then for $(7)$ it means that $D_{2}(s)=0$, which is well known in the literature [10. This also implies that $D_{2}(s)=0$ in (3). However, this is difficult to prove analytically. Instead, it is numerically verified that $D_{2}(s)=0$ in (3) by comparing it to finite difference simulations with boundary condition $T(\rho \gg 1)=0$. This shows that the error between the analytic and numerical simulations are small and that the error is decreasing with increasing density of the discretization grid. In addition, the functions $\Phi$ and $\Psi$ are numerically evaluated to study the behavior close to $\rho=\infty$, indicating again that this is the correct choice. Hence, it is concluded that $D_{2}(s)=0$ for a semi-infinite domain such that $(3)$ becomes

$$
\Theta(\rho, s)=D_{1}(s) e^{\lambda_{1} \rho} \Psi\left(\frac{\lambda_{2}}{\lambda_{2}-\lambda_{1}}, 1,\left(\lambda_{2}-\lambda_{1}\right) \rho\right) .
$$

In principle, $D_{1}(s)$ needs to be fixed by assuming a second boundary condition. However, it is common practice to express the solution in terms of the spatial logarithmic derivative of the amplitude $A$ and the spatial derivative of $\phi$, which can be calculated using the logarithmic temperature derivative $(\partial \Theta / \partial \rho) / \Theta$. Therefore, the temperature $\Theta$ is written in terms of its harmonic components, i.e., $\Theta=A \exp (i \phi)$, and the spatial derivative of the temperature is 
given by $\Theta^{\prime}=A^{\prime} \exp (i \phi)+i \phi^{\prime} A \exp (i \phi)$ such that

$$
\frac{\Theta^{\prime}}{\Theta}=\frac{A^{\prime}}{A}+i \phi^{\prime}
$$

Taking the logarithmic derivative of the right hand side of (8) results in, see [8]

$$
\frac{\Theta^{\prime}}{\Theta}=\lambda_{1}-\lambda_{2} \frac{\Psi\left(1+\frac{\lambda_{2}}{\lambda_{2}-\lambda_{1}}, 2,\left(\lambda_{2}-\lambda_{1}\right) \rho\right)}{\Psi\left(\frac{\lambda_{2}}{\lambda_{2}-\lambda_{1}}, 1,\left(\lambda_{2}-\lambda_{1}\right) \rho\right)},
$$

which will be used to derive an explicit relationship to identify $\chi$ in the presence of $V$ and $\tau_{i n v}$. If $V=0$, then 10 simplifies to

$$
\frac{\Theta^{\prime}}{\Theta}=-z \frac{K_{1}(z \rho)}{K_{0}(z \rho)}
$$

with $z=\frac{1}{2}\left(\lambda_{2}-\lambda_{1}\right)$. This relationship is well known in the literature [10].

A common problem using the representations $(10)$ and $(11)$ is how to calculate the spatial derivatives $A^{\prime} / A$ and $\phi^{\prime}$ from the measured $A$ and $\phi$. In contrast to the slab-geometry case, where the spatial derivative can be explicitly calculated, see [1, for cylindrical geometry the spatial derivative cannot be easily calculated. On the other hand, it is possible to avoid the calculation of the spatial derivatives by directly expressing the transport coefficients and $\rho$ in terms of the measured amplitudes using the transfer function description [1, 11-13]. This description needs a second boundary condition to define $D_{1}(s)$, which basically defines the relationship between $A^{\prime} / A$ and $A$, and $\phi^{\prime}$ and $\phi$.

The most logical choice for a second boundary condition is $\Theta(\rho, s)=\Theta\left(\rho_{1}, s\right)$, because $\Theta\left(\rho_{1}, s\right)$ is measured. In addition, the domain on which the transport coefficients are analyzed cannot contain a source term, but still this domain should be limited by a boundary condition. Using the same steps as presented in [1], the transfer function from the temperature at point $\rho_{1}$ to the temperature at point $\rho_{2}$ is given by

$$
\frac{\Theta\left(\rho_{2}, s\right)}{\Theta\left(\rho_{1}, s\right)}=e^{\lambda_{1} \Delta \rho} \frac{\Psi\left(\frac{\lambda_{2}}{\lambda_{2}-\lambda_{1}}, 1,\left(\lambda_{2}-\lambda_{1}\right) \rho_{2}\right)}{\Psi\left(\frac{\lambda_{2}}{\lambda_{2}-\lambda_{1}}, 1,\left(\lambda_{2}-\lambda_{1}\right) \rho_{1}\right)},
$$

where the solution at a second measurement point $\rho_{2}>\rho_{1}$ is used as resulting temperature $\Theta\left(\rho_{2}\right)$. The transfer function description avoids the ambiguity of how to determine $A^{\prime} / A$ and $\phi^{\prime}$. On the other hand, from the transfer function description in cylindrical geometry it is not straightforward to derive explicit relationships for $\chi$. On the other hand, the transfer 
description can be used to validate the estimated coefficients by comparing the measured left hand side to the right hand side where the estimated $\chi, V$, and $\tau_{i n v}$ need to be substituted. In particular, as the transfer function can be derived for many different choices of boundary conditions, e.g., a Dirichlet boundary condition or a Robin boundary condition at the edge could be used. As such, it is possible to check if the semi-infinite domain assumption is valid. However, in this paper this will not be further investigated. In the next section, approximations are derived to estimate $\chi, V$, and $\tau_{i n v}$.

\section{DERIVATION OF EXPLICIT APPROXIMATIONS}

Here, three new methods are introduced to find approximations for determining the diffusivity in cylindrical geometry using (10) and (11). They are based on continued fractions, asymptotic expansions, and multiple harmonics. A great number of new approximations are derived in this paper. Therefore, in this section only the basic ideas are explained to increase the readability of the paper. The full derivations are summarized in the next section and their derivations can be found in the appendix.

\section{A. Continued fractions}

The solution to the Bessel Differential Equation and Confluent Hypergeometric Differential Equation can be found by substitution of a power series. This yields the recurrence relationship, which is used to calculate the infinite series representation of Bessel functions and Confluent Hypergeometric Functions. Moreover, if a fraction of these functions is studied the recurrence relationships and series representation can also be rewritten in terms of a Continued Fractions representation [14, 15]. By truncating this Continued Fraction the ratio of Bessel functions and Confluent Hypergeometric Functions can be approximated. Here, this approximation is inverted such that the transport coefficients can be directly calculated.

The use of a continued fraction to derive an explicit approximation for $\chi$ is best explained by means of an example. Therefore, consider (11) where for $V=0$ such that a new variable $z$ can be defined:

$$
z=\sqrt{\frac{3}{2} \frac{i \omega+\tau_{i n v}}{\chi}} .
$$


The Laplace variable is replaced by $s=i \omega$ as $s$ can only be measured on the imaginary axis. The Continued $J$-fraction from [14, 15] can be used to calculate the ratio

$$
-\frac{\frac{K_{1}(z \rho)}{K_{0}(z \rho)}=1+\frac{1}{2 z \rho}}{2 z \rho+2-\frac{(4 z \rho)^{-1}}{2 z \rho+4-\frac{9 / 4}{2 z \rho+6-\frac{64 / 4}{\ldots}}}} .
$$

This continued fraction can be truncated to find explicit approximations for $\chi$. The most simple truncation is

$$
\frac{\Theta^{\prime}}{\Theta}=-z\left(1+\frac{1}{2 z \rho}\right)
$$

This can be rewritten by substituting $(9)$ and rewriting in terms of $z$

$$
z=-\left(\frac{A^{\prime}}{A}+i \phi^{\prime}+\frac{1}{2 \rho}\right)
$$

Now by substituting (13) and squaring (16) it is possible to find $\chi$ and $\tau_{\text {inv }}$ using the imaginary part and real part

$$
\chi_{c}=\frac{3}{4} \frac{\omega}{\left(\frac{A^{\prime}}{A}+\frac{1}{2 \rho}\right) \phi^{\prime}}
$$

and

$$
\tau_{c}=\frac{\omega}{2}\left(\frac{\left(\frac{A^{\prime}}{A}+\frac{1}{2 \rho}\right)}{\phi^{\prime}}-\frac{\phi^{\prime}}{\left(\frac{A^{\prime}}{A}+\frac{1}{2 \rho}\right)}\right) .
$$

The index, here $c$, is used to distinguish between the different approximations. In [4] the same approximation is found based on a different method. However, unlike [4] using this method also the corresponding formula to calculate $\tau_{i n v}$ is found.

This method can also be used to find more accurate approximations by using more terms in the continued fraction.

In principle, it is possible to find an approximation of arbitrary accuracy. However, in practice solving for $\chi$ beyond third order polynomials in $z$ is too complicated. The reason is that finding zeros for a fourth order polynomial in $z$ is not straightforward. In addition, different solutions apply for different regions of interest, because the coefficients in the polynomials in $z$ depend on $A^{\prime} / A$ and $\phi^{\prime}$. Hence, mathematical bifurcations can occur. 
The continued fraction in (14) can also be truncated at a later stage, for the first three terms of (14) this results in

$$
\frac{\Theta^{\prime}}{\Theta}=-z+\frac{1}{2 \rho}+\frac{1}{\rho}\left(-\frac{1 / 4}{2 z \rho+2-\frac{9 / 4}{2 z \rho+4}}\right) .
$$

It can be rewritten in terms of $z$ resulting in the third order polynomial

$$
0=a_{3} z^{3}+a_{2} z^{2}+a_{1} z+a_{0}
$$

with coefficients

$$
\begin{gathered}
a_{3}=16 \rho^{3}, a_{2}=16 \frac{\Theta^{\prime}}{\Theta} \rho^{3}+56 \rho^{2}, \\
a_{1}=48 \frac{\Theta^{\prime}}{\Theta} \rho^{2}+45 \rho, \text { and } a_{0}=23 \frac{\Theta^{\prime}}{\Theta} \rho+7.5,
\end{gathered}
$$

where $\Theta^{\prime} / \Theta$ is given by $(9)$. The third order polynomial yields three solutions. However, generally only one solution can be used to determine $\chi$, because the argument of $z$ is situated in the domain $[0, \pi / 4]\left(\chi, \tau_{\text {inv }}\right.$, and $\omega$ are larger than zero $)$ and the other two are outside this domain. This has been numerically calculated and it turns out that the correct zero is given by

$$
z=-\frac{a_{2}}{3 a_{3}}-\frac{\sqrt[3]{2} p_{0}}{3 a_{3} p_{1}}+\frac{p_{1}}{3 \sqrt[3]{2} a_{3}},
$$

where $p_{0}=3 a_{1} a_{3}-a_{2}^{2}, p_{1}=\sqrt[3]{p_{2}+\sqrt{4 p_{0}^{3}+p_{2}^{2}}}$, and $p_{2}=-27 a_{0} a_{3}^{2}+9 a_{1} a_{2} a_{3}-2 a_{2}^{3}$. Now the solution is found by squaring and using the imaginary part and the real part of $z^{2}$

$$
\chi=\frac{3}{2} \frac{\omega}{\Im\left(z^{2}\right)} .
$$

The solution for the semi-infinite domain approximations of a third order polynomial, i.e., (20), is always given by (22). This is because the argument of $z$ of the correct solution must be situated in $[0, \pi / 4]$. This is difficult to proof, but has been numerically verified. This holds similarly for a second order polynomial in $z$. Hence, $\chi$ can always be found for such polynomials in $z$ using (23), which is based on (13). Therefore, in the appendix only the polynomials in terms of $z$ are given. The approximation for $\tau_{i n v}$ can also be found based on (13)

$$
\tau_{i n v}=\omega \frac{\Re\left(z^{2}\right)}{\Im\left(z^{2}\right)} .
$$


However, the damping $\tau_{i n v}$ is much more sensitive to errors due to mismodeling [1]. Therefore, they generally do not give reasonable values for the true damping. This holds also for approximations of the convectivity $V$, which are derived in the next sections. Nevertheless, they play an important role in the validation of the $\chi$ estimates. In the appendix, other continued fractions are used to find explicit approximations for $\chi$.

\section{B. Asymptotic expansions}

An alternative to continued fractions are asymptotic expansions. An asymptotic expansion of a function can be based on either a truncation of its series expansion or on an approximation of the defining Ordinary Differential Equation for the function [7, 8, 16]. The asymptotic expansions can be calculated for the Bessel functions and for the Confluent Hypergeometric Function of the second kind $\Psi$. Here, an approximation is derived based on (10) in terms of $\Psi$ and in the appendix the derivation of the approximation for $\chi$ based on Bessel functions of the second kind can be found.

In [8, 17] the asymptotic expansion for $x \approx 0$ of $\Psi(a, b, x)$ are given. Using this we find for $\left(\lambda_{1}-\lambda_{2}\right) \rho$ small

$$
\begin{gathered}
\Psi\left(a, 1,\left(\lambda_{2}-\lambda_{1}\right) \rho\right)= \\
-\frac{1}{\Gamma(a)}\left(\ln \left(\left(\lambda_{2}-\lambda_{1}\right) \rho\right)+\digamma(a)+2 \gamma\right),
\end{gathered}
$$

and

$$
\Psi\left(a+1,2,\left(\lambda_{2}-\lambda_{1}\right) \rho\right)=\frac{\left(\left(\lambda_{2}-\lambda_{1}\right) \rho\right)^{-1}}{\Gamma(a+1)},
$$

where $a=\lambda_{2} /\left(\lambda_{2}-\lambda_{1}\right), \gamma$ is the Euler-Mascheroni constant and $\Gamma$ is the gamma function. It should not be confused with the particle flux $\Gamma$, which is not considered in this paper. The logarithmic derivative of $\Gamma$ is the digamma function denoted by $\digamma$ (often also denoted as $\psi$ ). The logarithmic temperature derivative can be calculated by substituting (25) and (26) into 10 )

$$
\frac{\Theta^{\prime}}{\Theta}=\lambda_{1}+\lambda_{2} \frac{\frac{1}{\Gamma(a+1)}\left(\left(\lambda_{2}-\lambda_{1}\right) \rho\right)^{-1}}{\frac{1}{\Gamma(a)}\left(\ln \left(\left(\lambda_{2}-\lambda_{1}\right) \rho\right)+\digamma(a)+2 \gamma\right)} .
$$

This can be simplified using the property $\Gamma(a+1)=a \Gamma(a)$ and $a=\lambda_{1} /\left(\lambda_{2}-\lambda_{1}\right)$

$$
\frac{\Theta^{\prime}}{\Theta}=\lambda_{1}+\frac{1}{\rho\left(\ln \left(\left(\lambda_{2}-\lambda_{1}\right) \rho\right)+\digamma(a)+2 \gamma\right)} .
$$


The digamma function $\digamma(a)$ as function of $a$ and the appearance of $\lambda_{1}$ in and outside the logarithm pose important obstacles to find approximations for $\chi$. Therefore, two simplification steps are necessary. The asymptotic expansion is based on $x \approx 0$, which implies that $\rho \approx 0$. Thus, the term with $1 / \rho$ will dominate over $\lambda_{1}$, hence 28 can be simplified to

$$
\frac{\Theta^{\prime}}{\Theta}=\frac{1}{\rho\left(\ln \left(\left(\lambda_{2}-\lambda_{1}\right) \rho\right)+\digamma(a)+2 \gamma\right)} .
$$

However, if $V \neq 0$ the problem of $\digamma(a)$ remains.

In case $V=0, a=\frac{1}{2}$ such that $\digamma\left(\frac{1}{2}\right)=-2 \ln (2)-\gamma$ resulting in

$$
\frac{\Theta^{\prime}}{\Theta}=\frac{1}{\rho\left(\ln \left(\left(\lambda_{2}-\lambda_{1}\right) \rho\right)-2 \ln (2)+\gamma\right)},
$$

rearranging yields

$$
\lambda_{2}-\lambda_{1}=\frac{1}{\rho} \exp \left(\frac{1}{\frac{\Theta^{\prime}}{\Theta} \rho}+2 \ln (2)-\gamma\right)
$$

such that

$$
\frac{1}{2}\left(\lambda_{2}-\lambda_{1}\right)=\frac{\gamma_{1}}{\rho} \exp \left(\left(\frac{\Theta^{\prime}}{\Theta} \rho\right)^{-1}\right),
$$

where $\gamma_{1}=2 \exp (-\gamma)$, which should be used with high accuracy in the calculations. The approximation in terms of $z$ is given by

$$
z=\frac{\gamma_{1}}{\rho} \exp \left(\left(\frac{\Theta^{\prime}}{\Theta} \rho\right)^{-1}\right)
$$

for which the solution in terms of $\chi$ and $\tau_{i n v}$ are given in 23 and (24). Note that in this special case $\chi$ and $\tau_{i n v}$ can also be expressed in terms of geometrical functions

$$
\chi_{A E \Psi}=\frac{3}{2} \omega \frac{\rho^{2}}{\gamma_{1}^{2}}\left(\exp \left(\frac{2}{\rho} \frac{A^{\prime} / A}{\left|\Theta^{\prime} / \Theta\right|^{2}}\right) \sin \left(-\frac{2}{\rho} \frac{\phi^{\prime}}{\left|\Theta^{\prime} / \Theta\right|^{2}}\right)\right)^{-1},
$$

and

$$
\tau_{A E \Psi}=\omega\left(\tan \left(-\frac{2}{\rho} \frac{\phi^{\prime}}{\left|\Theta^{\prime} / \Theta\right|^{2}}\right)\right)^{-1} .
$$

In the appendix, also a similar relationship is derived based on the asymptotic expansions of Bessel functions. This approximation only differs from 34 by the factor $\gamma_{1}$. The comparison of the approximations with and without $\gamma_{1}$ shows that using the factor $\gamma_{1}$ improves the result, thus only $(33)$ is presented here.

Due to the digamma function it is not possible when $V \neq 0$ to rewrite 29 such that the diffusivity $\chi$ can be estimated explicitly. Therefore, another approximation step is 
introduced when $V \neq 0$ by replacing $\digamma(a)=\digamma\left(\frac{1}{2}\right)$. Then, it is still possible to estimate $\chi$ well in cases where $a \approx \frac{1}{2}$, e.g., $V \approx 0$ or $\ln \left(\left(\lambda_{2}-\lambda_{1}\right) \rho\right) \gg \digamma(a)$. The reason is that $V$ is also contained in $\lambda_{2}-\lambda_{1}$. In that case (29) is expressed as

$$
\left(\frac{V}{2 \chi}\right)^{2}+\frac{3}{2} \frac{\tau_{i n v}}{\chi}+\frac{3}{2} \frac{i \omega}{\chi}=\frac{\gamma_{1}^{2}}{\rho^{2}} \exp \left(\left(\frac{\Theta^{\prime}}{\Theta} \rho\right)^{-1}\right)^{2} .
$$

Interestingly, the estimate (34) for the diffusivity $\chi$ does not change as the imaginary part only contains $\chi$. If $\tau_{\text {inv }}=0$ in $(36)$, then

$$
\left|V_{A E \Psi}\right|=\frac{3}{2} 2 \omega \frac{\sqrt{\Re\left\{z^{2}\right\}}}{\Im\left\{z^{2}\right\}} .
$$

However, the possibility to estimate $V$ in practice using (37) is questionable. The combination of $V$ and $\tau_{i n v}$ cannot be calculated using this approximation.

\section{Multiple harmonics}

Every harmonic fixes two degrees of freedom, which means in practice that either $\chi$ and $\tau_{i n v}$ or $\chi$ and $V$ can be estimated if only one harmonic is used. Therefore, to estimate $\chi, V$, and $\tau_{\text {inv }}$ together, it is necessary to use at least two harmonics. In [1] an approximation is derived based on slab-geometry. Here, an approximation is derived based on the extended model in [4]

$$
\chi=\frac{3}{4} \omega\left(\left(\frac{A^{\prime}}{A}+\frac{1}{2 \rho}+\frac{n^{\prime}}{2 n}+\frac{\chi^{\prime}}{2 \chi}+\frac{V}{2 \chi}\right) \phi^{\prime}+\frac{\phi^{\prime \prime}}{2}\right)^{-1}
$$

This relation is derived by substituting $\Theta=A \exp (i \phi)$ and its spatial derivatives $\Theta^{\prime}$ and $\Theta^{\prime \prime}$ into the Laplace transform of (1), which also includes the density (assumed constant outside this subsection). Generally, this model is simplified by assuming constant $\chi$ such that $\chi^{\prime} / 2 \chi=0$. In addition, it is assumed that $V=0$ and $\phi^{\prime \prime}=0$. However, it can be shown, by taking the spatial derivative of $(10)$, that $\phi^{\prime \prime} \neq 0$. On the other hand, without this assumption the relationship (38) cannot be used without approximating $\phi^{\prime \prime}$ directly.

In this paper, only the assumption $\phi^{\prime \prime}=0$ is retained, but by using two harmonics the term $\frac{\chi^{\prime}}{2 \chi}+\frac{V}{2 \chi}$ can be eliminated. As such, it is possible to calculate $\chi, V$, and $\tau_{i n v}$. This should make it possible to identify $\chi$ under influence of $V$ and $\tau_{i n v}$, accepting errors caused by the $\phi^{\prime \prime}=0$ assumption. Therefore, rewrite 38 in terms of the unknown quantities

$$
\chi\left(\frac{A^{\prime}}{A}+\frac{1}{2 \rho}+\frac{n^{\prime}}{2 n}\right)-\frac{3}{4} \frac{\omega}{\phi^{\prime}}=-\chi\left(\frac{\chi^{\prime}}{2 \chi}+\frac{V}{2 \chi}\right) .
$$


This gives for two harmonics $\omega_{1}$ and $\omega_{2}$ at the same $\rho$

$$
\begin{gathered}
\chi\left(\frac{A_{\omega_{1}}^{\prime}}{A_{\omega_{1}}}+\frac{1}{2 \rho}+\frac{1}{2} \frac{n^{\prime}}{n}\right)-\frac{3}{4} \frac{\omega_{1}}{\phi_{\omega_{1}}^{\prime}}= \\
\chi\left(\frac{A_{\omega_{2}}^{\prime}}{A_{\omega_{2}}}+\frac{1}{2 \rho}+\frac{1}{2} \frac{n^{\prime}}{n}\right)-\frac{3}{4} \frac{\omega_{2}}{\phi_{\omega_{2}}^{\prime}},
\end{gathered}
$$

where the short-hand notation $\phi_{\omega_{2}}^{\prime}=\phi^{\prime}\left(\omega_{2}\right)$ and $A_{\omega_{2}}^{\prime} / A_{\omega_{2}}=A^{\prime}\left(\omega_{2}\right) / A\left(\omega_{2}\right)$ is used. Solving 40) yields

$$
\chi_{c 2 H}=\frac{3}{4} \frac{\frac{\omega_{1}}{\phi^{\prime}\left(\omega_{1}\right)}-\frac{\omega_{2}}{\phi^{\prime}\left(\omega_{2}\right)}}{\left(\frac{A^{\prime}}{A}\left(\omega_{1}\right)-\frac{A^{\prime}}{A}\left(\omega_{2}\right)\right)} .
$$

Note that 41 is insensitive to density gradients. The convectivity $V$ is found by rewriting (39) and next to assuming $\phi^{\prime \prime}=0$ also assuming $\chi^{\prime}=0$

$$
V_{c 2 H}=-2\left(\frac{A^{\prime}}{A}+\frac{1}{2 \rho}+\frac{1}{2} \frac{n^{\prime}}{n}\right) \chi_{c 2 H}+\frac{3}{2} \frac{\omega}{\phi^{\prime}},
$$

where $\omega$ can be freely chosen, but $\omega_{1}$ is preferable. However, calculating the damping is more difficult. Therefore, after the substitution of $\Theta^{\prime}$ and $\Theta^{\prime \prime}$ into (1) the real part should be considered

$$
\begin{gathered}
\chi\left(\frac{A^{\prime \prime}}{A}-\left(\phi^{\prime}\right)^{2}\right)=\frac{3}{2} \tau_{i n v}-\left(\frac{1}{\rho} V+V^{\prime}+\frac{n^{\prime}}{n} V\right) \\
-\left\{\frac{1}{\rho} \chi+\frac{n^{\prime}}{n} \chi+V\right\} \frac{A^{\prime}}{A} .
\end{gathered}
$$

This can be rewritten in terms of $\tau_{i n v}$

$$
\begin{gathered}
\tau_{c 2 H}=\frac{2}{3} \chi\left(\left(\frac{A^{\prime \prime}}{A}-\left(\phi^{\prime}\right)^{2}\right)\right. \\
\left.+\left(\frac{1}{\rho} \frac{V}{\chi}+\frac{V^{\prime}}{\chi}+\frac{n^{\prime}}{n} \frac{V}{\chi}\right)+\left\{\frac{1}{\rho}+\frac{n^{\prime}}{n}+\frac{V}{\chi}\right\} \frac{A^{\prime}}{A}\right) .
\end{gathered}
$$

After the substitution of $(39)$ it yields

$$
\begin{aligned}
& \tau_{c 2 H}=\frac{2}{3} \chi\left(\left(\frac{A^{\prime \prime}}{A}-\left(\phi^{\prime}\right)^{2}\right)+\left(\frac{1}{\rho} \frac{V}{\chi}+\frac{V^{\prime}}{\chi}+\frac{n^{\prime}}{n} \frac{V}{\chi}\right)\right. \\
& \left.+\left\{\frac{1}{\rho}+\frac{n^{\prime}}{n}+\left(\frac{3}{4} \frac{\omega}{\phi^{\prime} \chi}-2\left(\frac{A^{\prime}}{A}+\frac{1}{2 \rho}+\frac{n^{\prime}}{2 n}\right)\right)\right\} \frac{A^{\prime}}{A}\right) .
\end{aligned}
$$


In this equation, still two unknowns are present, thus in the spirit of neglecting $\phi^{\prime \prime}, \frac{\partial}{\partial \rho}\left(\frac{A^{\prime}}{A}\right)$ is also neglected. In addition, the standard assumption of $V$ is constant in space is used such that $V^{\prime}=0$. This results in the following relationship

$$
\begin{gathered}
\tau_{c 2 H}=\frac{2}{3} \chi\left(\left(\frac{A^{\prime}}{A}\right)^{2}-\left(\phi^{\prime}\right)^{2}+\left(\frac{1}{\rho} \frac{V}{\chi}+\frac{n^{\prime}}{n} \frac{V}{\chi}\right)\right. \\
\left.+\left\{\frac{1}{\rho}+\frac{n^{\prime}}{n}+\left(\frac{3}{4} \frac{\omega}{\phi^{\prime} \chi}-2\left(\frac{A^{\prime}}{A}+\frac{1}{2 \rho}+\frac{n^{\prime}}{2 n}\right)\right)\right\} \frac{A^{\prime}}{A}\right) .
\end{gathered}
$$

The calculation of $\tau_{i n v}$ is based on a complex relationship. In addition, assuming $\phi^{\prime \prime}=0$ and $\frac{\partial}{\partial \rho}\left(\frac{A^{\prime}}{A}\right)=0$ introduces additional errors when estimating $\chi, V$, and $\tau_{i n v}$. This influences the approximations $\tau_{c 2 H}$ and $V_{c 2 H}$ more significantly and hence are not so useful in practice.

It may seem that assuming $\chi$ constant is no longer necessary by eliminating $\chi^{\prime}$ in (41). However, if $\chi^{\prime}$ is non-zero it also modifies $\phi^{\prime \prime}$. As such, it is questionable if better estimates of $\chi$ can be achieved. The density gradient is still included here to show that it is contained. However, in this paper the density is assumed constant such that $n^{\prime} / n=0$.

The methods of continued fractions, asymptotic expansions, and multiple harmonics are used to derive many different explicit approximations of $\chi$. They are all approximations and all have a different region in which their approximation is good and regions in which it is poor. The other explicit approximations are presented in the appendix, which together with the approximations presented here are summarized and compared in the next section.

\section{OUTWARD SOLUTIONS}

In this section, the explicit approximations for $\chi$ in a semi-infinite cylindrical geometry with constant spatial parameter dependencies are summarized and compared. The compared approximations, based on (10) and (11), are presented in tabularized form in this section. In addition, the slab-geometry formula's in [1] are also considered here.

This section consists of four parts: the overview table with all the derived approximations; a presentation and discussion on the selection of the best approximations when only $\chi$ is considered; a similar discussion when $\chi$ and $\tau_{\text {inv }}$ are considered $(V=0)$; and when $\chi, V$, and $\tau_{i n v}$ are considered. The comparison is based on five parameters $\left(\rho, \omega, \chi, V\right.$, and $\left.\tau_{i n v}\right)$ and is presented in terms of normalized transport coefficients, i.e., $\bar{\chi}=\chi / \omega, \bar{V}=V / \omega$, and 
$\bar{\tau}_{i n v}=\tau_{i n v} / \omega$. In case two harmonics are necessary, $A^{\prime} / A\left(\omega_{1}\right), A^{\prime} / A\left(\omega_{2}\right), \phi^{\prime}\left(\omega_{1}\right)$, and $\phi^{\prime}\left(\omega_{2}\right)$ are calculated using $\omega_{1}=\omega$ and $\omega_{2}=2 \omega$ corresponding to the first and second harmonic.

\section{A. Overview of possible explicit approximations}

In Table $\mathrm{I}$ the derived approximations to calculate $\chi$ from Section III and the appendix are summarized including some relationships from the literature. The following notation is used to express approximations based on continued fractions $\chi_{K j 3}: K$ denotes the Bessel function of the second kind, small $j$ denotes the $J$-fraction, and 3 denotes the approximation order (truncation). The approximations derived from asymptotic expansions are denoted as follows: $\chi_{A E \Psi}$, where $A E$ means Asymptotic Expansion and $\Psi$ the Confluent Hypergeometric Function of the second kind on which it is based. 


\begin{tabular}{|c|c|c|c|c|c|}
\hline & $\chi$ & Equation for $\chi$ & Equation for $V$ & Equation for $\tau_{i n v}$ & ref. \\
\hline & $\chi_{s 1}$ & $\frac{3}{4} \frac{\omega}{\left(\phi^{\prime}\right)^{2}}$ & 0 & 0 & 18 \\
\hline $\mathrm{s}$ & $\chi_{s 2}$ & $\frac{3}{4} \frac{\omega}{\left(A^{\prime} / A\right)^{2}}$ & 0 & 0 & 19. \\
\hline 1 & $\chi_{s 3}$ & $\frac{3 \omega}{\left(A^{\prime} / A+\phi^{\prime}\right)^{2}}$ & 0 & 0 & 20 \\
\hline $\mathrm{a}$ & $\chi_{s 4}$ & $\frac{3}{4} \frac{\omega}{\left(A^{\prime} / A\right) \phi^{\prime}}$ & 0 & $\frac{\omega}{2}\left(\frac{A^{\prime} / A}{\phi^{\prime}}-\frac{\phi^{\prime}}{A^{\prime} / A}\right)$ & 21 \\
\hline \multirow[t]{3}{*}{$\mathrm{b}$} & $\chi_{V}$ & $\frac{3}{2} \frac{\omega \frac{A^{\prime}}{A}}{\left(\left(\frac{A^{\prime}}{A}\right)^{2}+\left(\phi^{\prime}\right)^{2}\right) \phi^{\prime}}$ & $\frac{3}{2} \frac{\omega\left(\left(\phi^{\prime}\right)^{2}-\left(\frac{A^{\prime}}{A}\right)^{2}\right)}{\left(\left(\frac{A^{\prime}}{A}\right)^{2}+\left(\phi^{\prime}\right)^{2}\right) \phi^{\prime}}$ & $-(1 \%+5 \%$ & 1 \\
\hline & \multirow[b]{2}{*}{$\chi_{\phi}$} & \multirow{2}{*}{$\frac{3}{4} \sqrt{\frac{\left(\omega_{1} \phi_{\omega_{2}}^{\prime}\right)^{2}-\left(\omega_{2} \phi_{\omega_{1}}^{\prime}\right)^{2}}{\phi_{\omega_{1}}^{\prime 2} \phi_{\omega_{2}}^{\prime 2}\left(\phi_{\omega_{1}}^{\prime 2}-\phi_{\omega_{2}}^{\prime 2}\right)}}$} & $-2 \chi \frac{A^{\prime}}{A}-\sqrt{\frac{C_{V}+\sqrt{C_{V}^{2}+36 \chi^{2} \omega^{2}}}{2}}$ & $\frac{C_{V}-V^{2}}{6 \chi}$ & \multirow{2}{*}{1} \\
\hline & & & \multicolumn{2}{|c|}{$C_{V}=\frac{9}{4} \omega^{2}\left(\phi^{\prime}\right)^{-2}-4 \chi^{2}\left(\phi^{\prime}\right)^{2}$} & \\
\hline $\mathrm{c}$ & $\chi_{c}$ & $\frac{3}{4} \frac{\omega}{\phi^{\prime}\left(\frac{A^{\prime}}{A}+\frac{1}{2 \rho}\right)}$ & 0 & $\frac{\omega}{2}\left(\frac{\left(\frac{A^{\prime}}{A}+\frac{1}{2 \rho}\right)}{\phi^{\prime}}-\frac{\phi^{\prime}}{\left(\frac{A^{\prime}}{A}+\frac{1}{2 \rho}\right)}\right)$ & [4] \\
\hline $\mathrm{y}$ & $\chi_{K j 1 A}$ & $\frac{3}{4} \frac{\omega}{\left(\frac{A^{\prime}}{A}+\frac{1}{2 \rho}\right)^{2}}$ & 0 & 0 & 54 \\
\hline 1 & $\chi_{z}$ & $\frac{3}{2} \frac{\omega}{\Im\left(z^{2}\right)}$ & 0 & $\omega \frac{\Re\left(z^{2}\right)}{\Im\left(z^{2}\right)}$ & 23 \\
\hline & $\chi_{c 2 H}$ & $\frac{3}{4} \frac{\omega_{1} / \phi_{\omega_{1}}^{\prime}-\omega_{2} / \phi_{\omega_{2}}^{\prime}}{\left(A_{\omega_{1}}^{\prime} / A_{\omega_{1}}-A_{\omega_{2}}^{\prime} / A_{\omega_{2}}\right)}$ & $-2 \chi\left(\frac{A^{\prime}}{A}+\frac{1}{2 \rho}\right)+\frac{3}{2} \frac{\omega}{\phi^{\prime}}$ & $\frac{2}{3} \chi\left(\left(\frac{A^{\prime}}{A}\right)^{2}-\left(\phi^{\prime}\right)^{2}+\frac{1}{\rho} \frac{A^{\prime}}{A}+\frac{V}{\chi} \frac{A^{\prime}}{A}\right.$ & 41 \\
\hline
\end{tabular}

Table I: Overview of approximations for $\chi$ based on a semi-infinite domain where heat waves are traveling towards the edge in a cylindrical geometry and slab geometry. From left to right, the columns denote: the geometry on which it is based; the approximation of $\chi$ either explicit or in terms of $z$ in which case Table II gives the relationship for $z$; and the equations for $V$ and $\tau_{i n v}$. In the last column, the reference of the origin of the equation are given. The short-hand notations $\phi^{\prime}\left(\omega_{1}\right)=\phi_{\omega_{1}}^{\prime}$ and $\frac{A^{\prime}\left(\omega_{1}\right)}{A\left(\omega_{1}\right)}=\frac{A_{\omega_{1}}^{\prime}}{A_{\omega_{1}}}$ are used. In slab-geometry the spatial derivatives are linear such that between two measurement points $\rho_{1}$ and $\rho_{2}$ the spatial derivatives are defined as $A^{\prime} / A=\ln \left(A_{2} / A_{1}\right) /\left(\rho_{2}-\rho_{1}\right)$ and $\phi^{\prime}=\left(\phi_{2}-\phi_{1}\right) /\left(\rho_{2}-\rho_{1}\right)$, where $\rho_{2}>\rho_{1}$, see [1] for details.

Many equations can also be expressed using polynomials in terms of $z, \Theta^{\prime} / \Theta$, and $\rho$ to directly calculate $\chi$ and $\tau_{i n v}$. Therefore, Table II gives the coefficients of these polynomials with their solutions to calculate $z$. Note that the approximations for $\tau_{i n v}$ and $V$ in Table I can show large deviations from the true values. Therefore, they should be used carefully, and in this paper they are only used in the validation process. 


\begin{tabular}{|c|c||c|}
\hline$\chi_{z}$ & Equation for $z$ & Eq. \\
\hline$\chi_{A E K}$ & $z=\rho^{-1} \exp \left(\left(\rho \frac{\Theta^{\prime}}{\Theta}\right)^{-1}\right)$ & 34 \\
\hline$\chi_{A E \Psi}$ & $z=\frac{\gamma_{1}}{\rho} \exp \left(\left(\frac{\Theta^{\prime}}{\Theta} \rho\right)^{-1}\right)$ & 33 \\
\hline \hline$\chi_{K c 2}$ & $b_{2}=4 \rho, \quad b_{1}=3+4 \frac{\Theta^{\prime}}{\Theta} \rho, \quad b_{0}=\frac{\Theta^{\prime}}{\Theta}$ & 48 \\
\hline$\chi_{K j 2}$ & $b_{2}=8 \rho^{2}, \quad b_{1}=8 \rho^{2} \frac{\Theta^{\prime}}{\Theta}+4 \rho, b_{0}=8 \frac{\Theta^{\prime}}{\Theta} \rho-3$ & 48 \\
\hline \multicolumn{3}{|c|}{$z=\left(-b_{1}+\sqrt{b_{1}^{2}-4 b_{0} b_{2}}\right) / b_{2}$} \\
\hline$\chi_{K j 3}$ & $a_{3}=16 \rho^{3}, \quad a_{2}=16 \frac{\Theta^{\prime}}{\Theta} \rho^{3}+56 \rho^{2}$ & \\
\hline$\chi_{K c 5}=48 \frac{\Theta^{\prime}}{\Theta} \rho^{2}+45 \rho, \quad a_{0}=23 \frac{\Theta^{\prime}}{\Theta} \rho+7.5$ & 21 \\
\hline$\chi_{3}=16 \rho^{2}, \quad a_{2}=\left(36 \rho+16 \rho^{2} \frac{\Theta^{\prime}}{\Theta}\right)$ & \\
\hline \multicolumn{2}{|c|}{$a_{2}=-27 a_{0} a_{3}^{2}+9 a_{1} a_{2} a_{3}-2 a_{2}^{3}, p_{0}=3 a_{1} a_{3}-a_{2}^{2}$} \\
$p_{1}=\sqrt[3]{p_{2}+\sqrt{4 p_{0}^{3}+p_{2}^{2}}, z=\frac{1}{a_{3}}\left(-\frac{a_{2}}{3}-\frac{\sqrt[3]{2} p_{0}}{3 p_{1}}+\frac{p_{1}}{3 \sqrt[3]{2}}\right)}$ \\
\hline
\end{tabular}

Table II: Overview of approximations for $\chi$ in terms of $z$ for heat waves traveling towards the edge in a cylindrical geometry where an infinite domain is assumed. This table denotes the coefficients to calculate $z$ using $\Theta^{\prime} / \Theta=A^{\prime} / A+i \phi^{\prime}$ and $\rho$, which is used to calculate $\chi=\frac{3}{2} \omega / \Im\left(z^{2}\right)$ and $\tau_{\text {inv }}=\omega \Re\left(z^{2}\right) / \Im\left(z^{2}\right)$ based on the definition of $z$ in $(13)$. The equation numbers refer either to Section III or the appendix.

The different approximations for $\chi$ in this table are compared in the next subsection.

\section{B. Diffusivity only}

The comparison for $\chi$ only $\left(V=0\right.$ and $\left.\tau_{i n v}=0\right)$ is made based on a large number of possibilities of $\chi, \omega$, and $\rho$.

There are different methods to find $\phi^{\prime}$ and $A^{\prime} / A$, which can influence the results significantly. Therefore, to avoid any ambiguity on how the derivatives $\phi^{\prime}$ and $A^{\prime} / A$ should be approximated and how they affect the comparison, the true derivatives are used instead, which are generated by (11). The most interesting and best approximations are shown in Fig. 1 in terms of the relative error with respect to the true diffusivity $\chi$. 


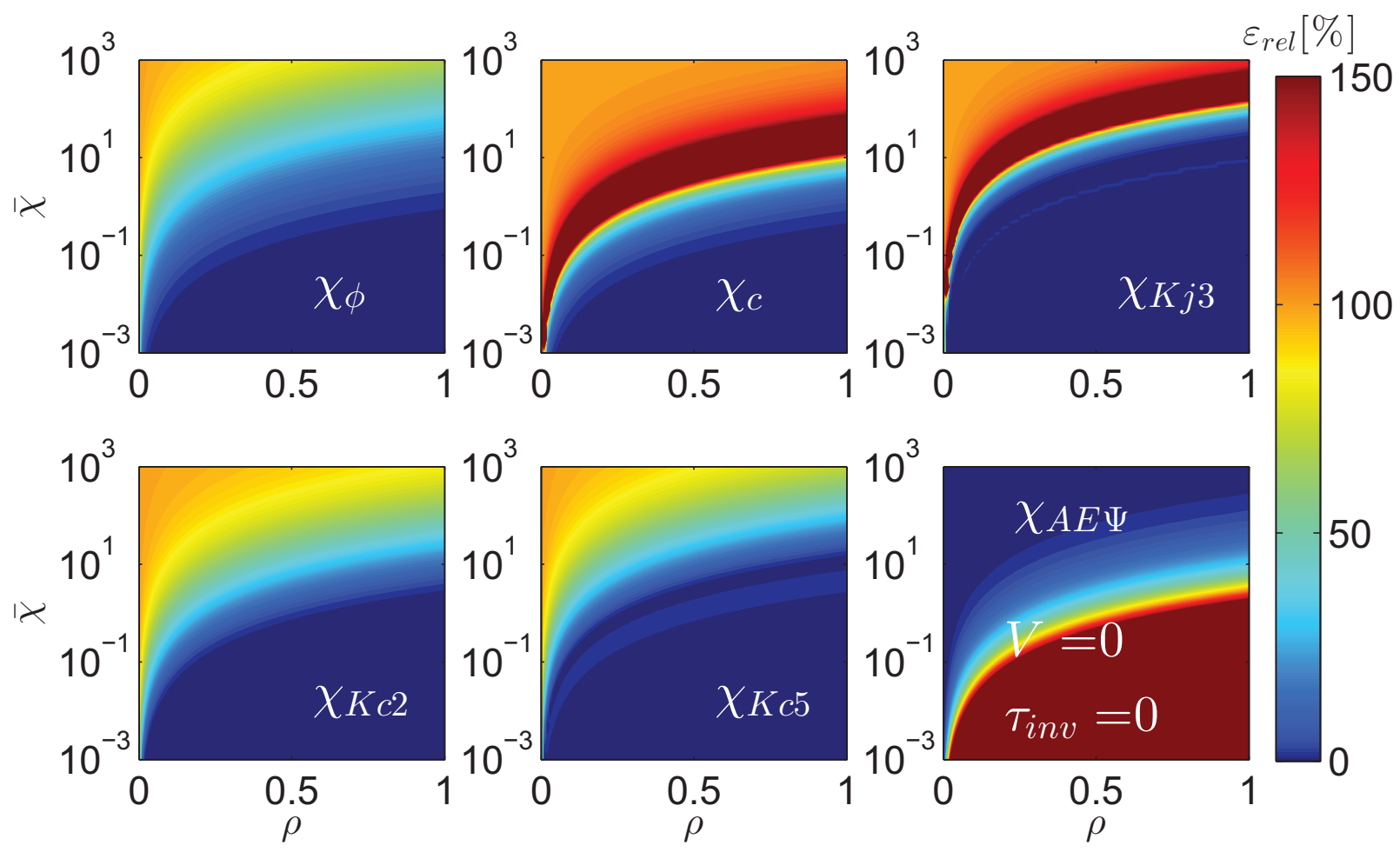

Figure 1: Comparison between the different relative errors of the $\chi$ estimates for a large range of $\bar{\chi}=\chi / \omega$ and $\rho$. The relative error is defined as $\varepsilon_{r e l}=100 \times \frac{|\chi-\chi e s t|}{\chi}[\%]$, where $\chi_{\text {est }}$ is either $\chi_{\phi}(2$ harmonics are used, which are $\omega_{1}=\omega$ and $\left.\omega_{2}=2 \omega\right), \chi_{c}, \chi_{K j 3}, \chi_{K c 2}, \chi_{K c 5}$, and $\chi_{A E \Psi}$ from Table [1] This comparison is based on a cylindrical geometry using an infinite domain boundary condition with $\chi$ and $V=\tau_{i n v}=0$, where the heat waves travel outwards. The darkest blue represents $\varepsilon_{\text {rel }}<1 \%$ and the darkest red represents all $\varepsilon_{\text {rel }}>150 \%$.

The approximations $\chi_{\phi}, \chi_{c}, \chi_{K j 3}, \chi_{K c 2}$, and $\chi_{K c 5}$ are basically extensions of the slabgeometry case as they estimate $\chi$ well if the ratio $\omega \rho / \chi$ is large. Perturbations with large $\omega$ or small $\chi$ penetrate less deep. This also holds for errors and the influence of cylindrical geometry, which allows $\chi$ to be estimated well by $\chi_{\phi}, \chi_{c}, \chi_{K j 3}, \chi_{K c 2}$, and $\chi_{K c 5}$.

It is important to remember that $\chi_{\phi}$ is based on two phases, making it less comparable to the other approximations. In $\chi_{c}$ and $\chi_{K j 3}$ large relative errors are observed for small $\rho$ and large $\bar{\chi}$, which can be understood by considering $\chi_{c}$ in (17). This large error is caused by $(2 \rho)^{-1}$ in $\chi_{c}$, which over compensates resulting in a higher estimated diffusivity [4]. In addition, $A^{\prime} / A$ and $\phi^{\prime}$ are negative quantities for heat waves traveling towards the edge, hence the sum of $(2 \rho)^{-1}$ and $A^{\prime} / A$ results in zero at the center of the dark red area. On the other hand, the large errors are more difficult to show intuitively for the approximation 
$\chi_{K j 3}$. The approximation $\chi_{K j 3}$ is more accurate and extends the region in which $\chi$ can be estimated well, which is a logical consequence of taking more terms in the continued fraction before truncation. Clearly, $\chi_{K c 2}$ and $\chi_{K c 5}$ do not suffer from the zero crossing causing the large errors in $\chi_{c}$ and $\chi_{K j 3}$. The region in which $\chi$ is estimated well by $\chi_{K c 5}$ is slightly larger than $\chi_{c}$ and smaller than $\chi_{K j 3}$.

Generally, $\chi_{K j 3}$ gives the best approximation of $\chi$ except when $\omega \rho / \chi$ is small. In that region the asymptotic expansions play an important role. They estimate $\chi$ well for $z \rho \approx 0$, which can be clearly seen in Fig. 1 for $\chi_{A E \Psi}$, which gave the best result of the two asymptotic expansions. This also means that if $\chi_{K j 3}$ and $\chi_{A E \Psi}$ are combined almost the entire region of $\chi$ is estimated well.

\section{Diffusivity and damping only}

There are a number of suitable approximations to determine $\chi$ in the presence of $\tau_{i n v}$. However, it suffices with a minimal loss of accuracy to use the approximations $\chi_{K j 3}$ and $\chi_{A E \Psi}$ to estimate $\chi$ in the region of interest, which is shown in Fig. 2 . 


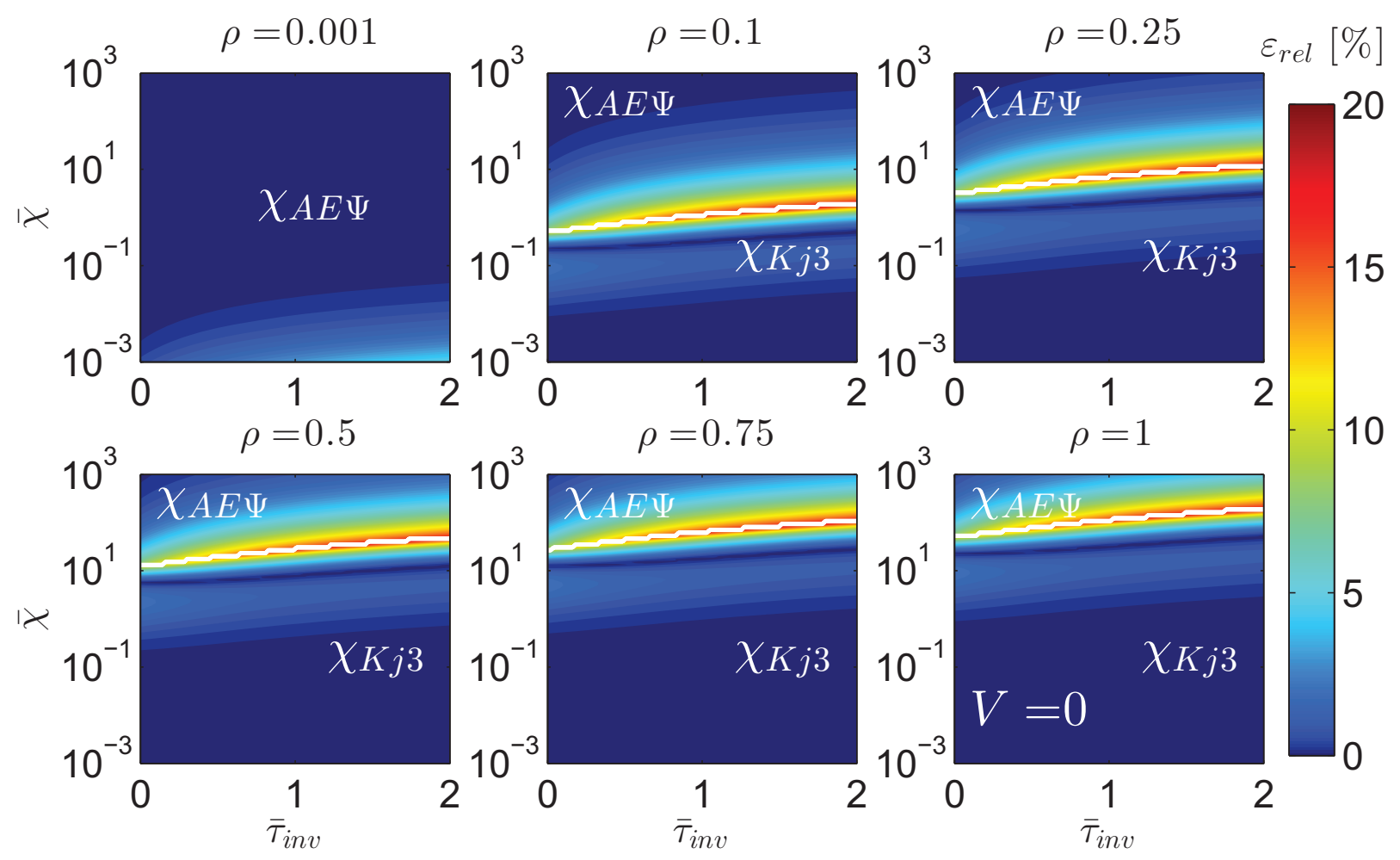

Figure 2: Relative error of the $\chi$ estimates for the combination of $\chi_{K j 3}$ and $\chi_{A E \Psi}$ presented for different $\bar{\chi}=\chi / \omega$ and $\bar{\tau}_{i n v}=\tau_{i n v} / \omega$ represented at a number of spatial locations $\rho$. The relative error is defined as $\varepsilon_{r e l}=100 \times \frac{\left|\chi-\chi_{e s t}\right|}{\chi}[\%]$. This figure combines the approximations $\chi_{K j 3}$ and $\chi_{A E \Psi}$ which take both $\chi$ and $\tau_{i n v}$ into account and are separated by the boundary represented by the white line (middle of the largest error). The resulting error is based on a cylindrical geometry using an infinite domain boundary condition with $\chi$ and $V=0$, where the heat waves travel outwards.

The approximations are presented at a limited number of spatial locations $\rho$. In order to have significant impact on the heat pulse propagation, $\tau$ should be of the order of the energy confinement time $\left(\tau_{e}\right)$, i.e., $1 \mathrm{~s}$ for JET or ITER. Therefore, the range of $\tau$ is chosen such that $0.5<\tau<\infty\left(\tau=\infty\right.$ meaning no damping), i.e., $0 \leqslant \tau_{i n v} \leqslant 2$. This range is the same for the normalized $\bar{\tau}_{\text {inv }}$ as the applicable range of $\omega$ is assumed $\omega>1[\mathrm{rad} / \mathrm{s}]$.

The white line shows the approximate boundary of the applicability of $\chi_{K j 3}$ and $\chi_{A E \Psi}$. This does not mean that $\chi_{K j 3}$ and $\chi_{A E \Psi}$ estimate $\chi$ with the highest accuracy compared to the other approximations in the presented region, but they have the largest region of approximation and are the most accurate in a large region. In addition, around the white line where the errors are largest $\chi_{K j 3}$ and $\chi_{A E \Psi}$ are the best approximations, which can also 
be understood by considering Fig. 1 again.

In general the effect of damping $\tau_{i n v}$ acts as a shift parameter and is not directly influenced by the cylindrical geometry [1]. However, these effects are also influenced by the approximation. This means that for large $\tau_{i n v}$ the regions in which $\chi$ are estimated well is extended for $\chi_{K j 3}$, and similarly for $\chi_{\phi}, \chi_{c}, \chi_{K c 2}$, and $\chi_{K c 5}$. On the other hand, it is reduced for $\chi_{A E \Psi}$, which is also shifted in the same direction.

The maximum relative error for the combination of $\chi_{A E \Psi}$ and $\chi_{K j 3}$ over the entire presented region is $\varepsilon_{r e l}<20 \%$. It is also important to note that in some regions $\chi_{K c 2}, \chi_{K c 5}$, and $\chi_{K j 2}$ give a slightly better approximation. In some isolated regions the other approximations than the previously mentioned approximations, give a better approximation than the two presented in Fig. 2, but generally with a comparable accuracy. Note that the absolute error for large $\bar{\chi}$ will be larger than $20 \%$.

\section{Diffusivity and convectivity with $\tau_{i n v}=0$ and $\tau_{i n v}=2$}

Most of the previously discussed approximations poorly estimate the diffusivity in the presence of convectivity. In principle, only four relationships are available to analyze $\chi$ for heat waves traveling outwards, i.e., $\chi_{V}, \chi_{\phi}, \chi_{A E \Psi}$, and $\chi_{c 2 H}$ (see Table I). It is unclear what a good range is for the parameter $\bar{V}=V / \omega$ except that it can also be negative. Therefore, an arbitrary choice for this range is made $-100 \leqslant \bar{V} \leqslant 100$.

It turns out that $\chi_{\phi}$ outperforms all other approximations except $\chi_{A E \Psi}$ in a more stronger cylindrical geometry. On the other hand, $\chi_{c 2 H}$ performs similar to $\chi_{\phi}$ both in error and the region in which it estimates $\chi$ well, making them almost interchangeable. Although they are both based on (the first) two harmonics, $\chi_{\phi}$ only uses the phase. Therefore, only $\chi_{\phi}$ and $\chi_{A E \Psi}$ are presented in Fig. 3 for $\tau_{i n v}=0$ and Fig. 4 for $\tau_{i n v}=2$. 


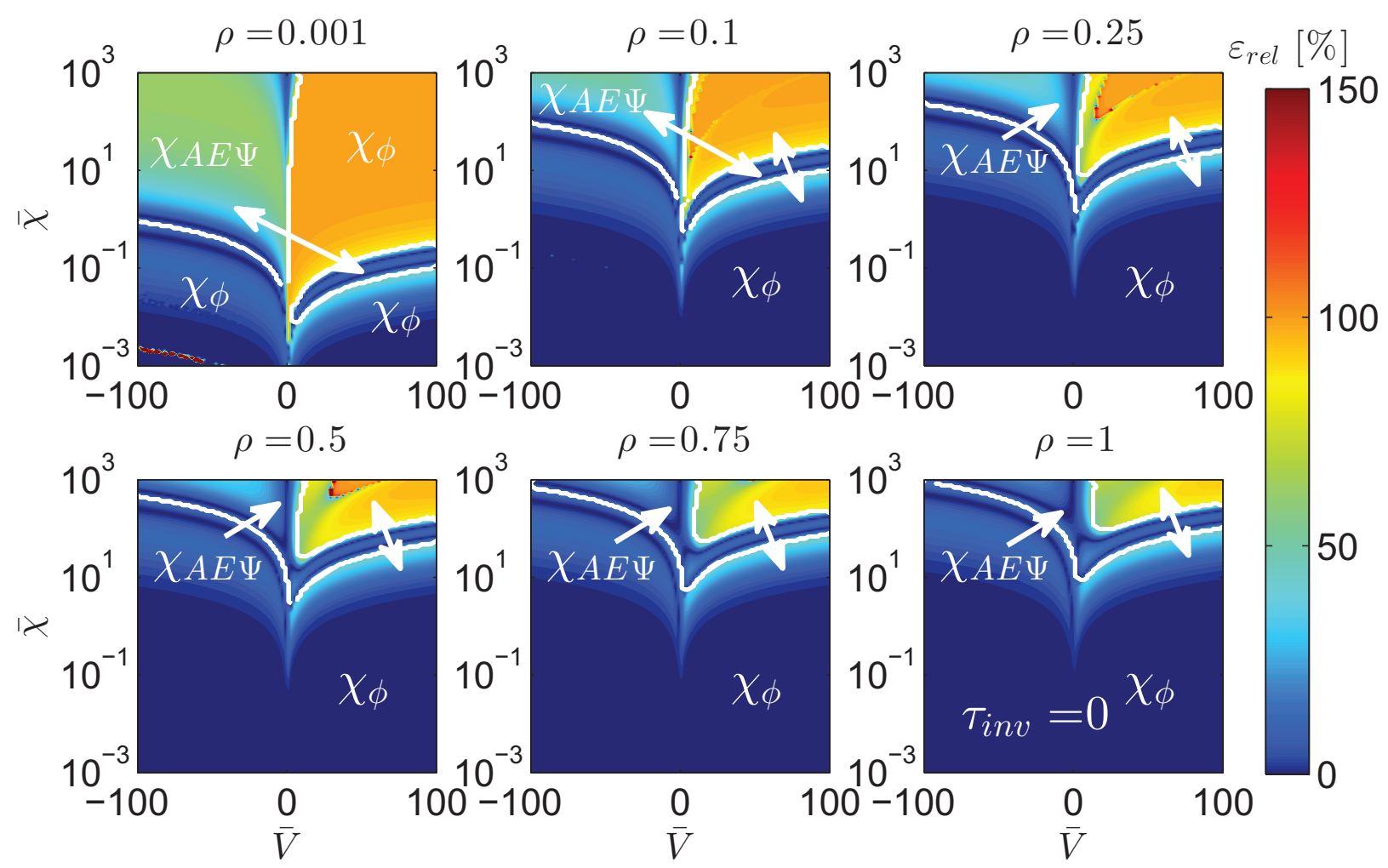

Figure 3: Relative error of the $\chi$ estimates for the combination of $\chi_{\phi}$ and $\chi_{A E \Psi}$ presented for different $\bar{\chi}=\chi / \omega$ and $\bar{V}=V / \omega$ represented at a number of spatial locations $\rho$. The relative error is defined as $\varepsilon_{\text {rel }}=100 \times \frac{\left|\chi-\chi_{\text {est }}\right|}{\chi}$ [\%]. The approximation regions of $\chi_{\phi}$ and $\chi_{A E \Psi}$ are separated by white lines. This comparison is based on a cylindrical geometry using an infinite domain boundary condition with $\tau_{i n v}=0$, where the heat waves travel outwards. The darkest blue represents $\varepsilon_{\text {rel }}<1 \%$ and the darkest red represents all $\varepsilon_{\text {rel }}>150 \%$. 


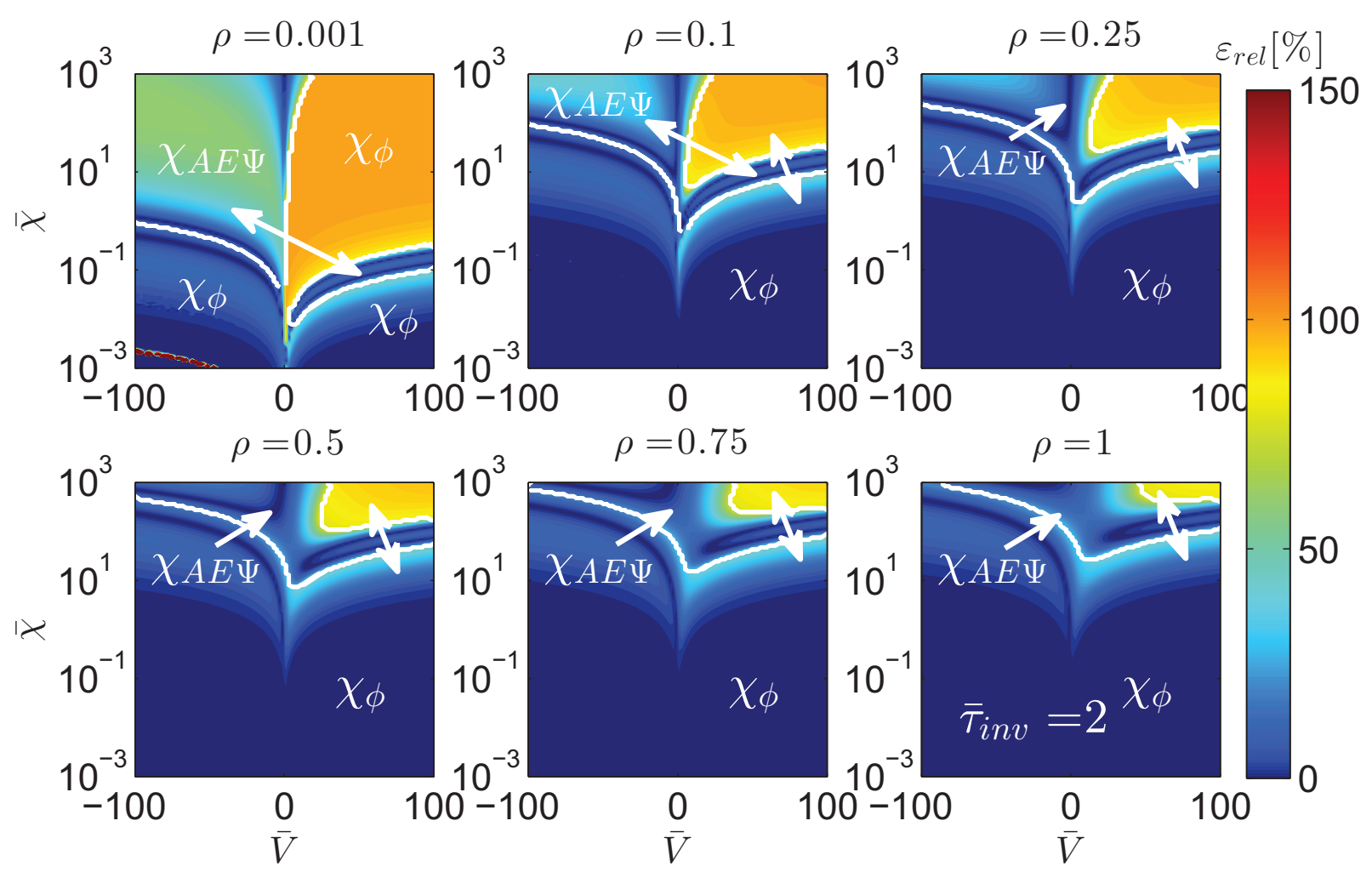

Figure 4: Relative error of the $\chi$ estimates for the combination of $\chi_{\phi}$ and $\chi_{A E \Psi}$ presented for different $\bar{\chi}=\chi / \omega$ and $\bar{V}=V / \omega$ represented at a number of spatial locations $\rho$. The relative error is defined as $\varepsilon_{r e l}=100 \times \frac{\left|\chi-\chi_{e s t}\right|}{\chi}[\%]$. The approximation regions of $\chi_{\phi}$ and $\chi_{A E \Psi}$ are separated by white lines. This comparison is based on a cylindrical geometry using an infinite domain boundary condition with $\bar{\tau}_{i n v}=2$, where the heat waves travel outwards. The darkest blue represents $\varepsilon_{r e l}<1 \%$ and the darkest red represents all $\varepsilon_{r e l}>150 \%$.

Fig. 3 and Fig. 4 show similar regions. In general $\chi$ can be estimated well for large $\rho$, but there is an area with higher errors. Although this area is influenced by the damping, its effect is rather small. In addition, there is a small region which has large errors $\left(\varepsilon_{\text {rel }}>150 \%\right)$ for large negative $V$ and for small $\rho$ and $\bar{\chi}$.

\section{E. Summary}

In this section many different approximations are introduced. For problems with convective velocity, $\chi_{\phi}$ and $\chi_{A E \Psi}$ should be used. Otherwise, $\chi_{A E \Psi}, \chi_{\phi}$, and $\chi_{K j 3}$ are the most important approximations. The other approximations can also be used. Although these 
other approximations are sometimes more accurate, their accuracy is in general comparable to the approximations given in Fig. 2 and Fig. 3. In Section $\mathrm{V}$ it is explained how to select the proper approximation based on the underlying models.

\section{CHOICE AND VALIDATION OF APPROXIMATIONS}

In the previous section many different approximations have been presented. The validity ranges of these approximations do not only depend on $\rho$ and $\omega$, but also on the unknown transport coefficients $\chi, V$, and $\tau_{i n v}$. This may seem to be a problem, but it is not. The reason is that the original models in terms of Bessel functions or Confluent Hypergeometric Functions are also available in $(10)$ and (11). They can be used to explicitly calculate estimates of the original $A^{\prime} / A$ and $\phi^{\prime}$ denoted by $\widehat{A^{\prime} / A}$ and $\widehat{\phi^{\prime}}$ given $\rho$ and the transport coefficients, which should approximately be the same.

The procedure to select the approximation for the cases with only $\chi$ and $\tau_{i n v}$ is shown through an example.

Example: Consider a measurement at $\rho=0.1$ where $A^{\prime} / A=-7.0593$ and $\phi^{\prime}=-3.5588$ with $\omega=50 \pi$ and assuming that $V=0$. Basically, two approximations are best tried first, i.e., $\chi_{K j 3}$ and $\chi_{A E \Psi}$. This results in $\chi_{K j 3}=7.8629, \tau_{K j 3}=-5.0253$ and $\chi_{A E \Psi}=13.1327$, $\tau_{A E \Psi}=72.4163$ using Table II and Table I. Clearly, the $\tau_{i n v}$ 's are erroneous. To test which is the proper approximation, substitute the values of $\chi$ and $\tau_{i n v}$ back into the original model, which in this case is 11 . This results in $\widehat{A^{\prime} / A}+\widehat{\phi}^{\prime} i=-6.8527-2.2746 i$ for $\chi_{K j 3}$, and $\widehat{A^{\prime} / A}+\widehat{\phi^{\prime}} i=-7.0377-3.6514 i$ for $\chi_{A E \Psi}$. It is immediately clear that $\chi_{K j 3}$ is the best approximation as $\widehat{A^{\prime} / A} \approx A^{\prime} / A$ and $\widehat{\phi^{\prime}} \approx \phi^{\prime}$.

It is also possible to use $\chi_{c}$ and $\chi_{K j 2}$ resulting in $\chi_{c}=10.7168, \tau_{c}=-90.2764$ and $\chi_{K j 2}=7.9655, \tau_{K j 2}=-18.1707$, with $\widehat{A^{\prime} / A}+\widehat{\phi^{\prime}} i=-5.9559-4.1495 i$ and $\widehat{A^{\prime} / A}+\widehat{\phi^{\prime}} i=$ $-6.8924-3.7907 i$, respectively. Again, $\chi_{K j 3}$ is closest to the true $A^{\prime} / A$ and $\phi^{\prime}$ and hence should be selected.

In this example, the true values are $\chi=8$ and $\tau_{i n v}=0.5$ such that actually $\chi_{K j 2}$ is closest to the true $\chi$. However, due to the much larger error in $\tau_{K j 2}$ the $\widehat{A^{\prime} / A}$ and $\widehat{\phi^{\prime}}$ deviate significantly from the true $A^{\prime} / A$ and $\phi^{\prime}$.

Hence, it is the combination of $\chi$ and $\tau_{i n v}$, which is tested. Nevertheless, this test gives a clear statement of which $\chi$ estimate is trustworthy. In most cases the exact $A^{\prime} / A=\widehat{A^{\prime} / A}=$ 
and $\phi^{\prime}=\widehat{\phi^{\prime}}$ are found with some small rounding error, unlike the example presented here.

Similarly, the approximation can be tested for cases of approximations for $\chi$ only or for problems with $\chi$ and $V$ only as only one harmonic is necessary. However, two harmonics are necessary for the mixed case of $\chi, V$, and $\tau_{i n v}$. This procedure is more difficult. It is still

possible to compare $\widehat{A^{\prime} / A}$ and $\widehat{\phi^{\prime}}$ to $A^{\prime} / A$ and $\phi^{\prime}$, but this comparison also depends on $A^{\prime} / A$ and $\phi^{\prime}$ of the second harmonic, which can also introduce a difference even if the estimated parameters are correct. Nevertheless, this comparison still gives valuable information on the quality of the approximation used. In case $\chi_{\phi}$ or $\chi_{V}$ are used, the transformation in [1] can be used to calculate $V$ and $\tau_{i n v}$.

If $V \neq 0$, then also an implementation of the Confluent Hypergeometric Function is required, which is not always available especially for complex valued arguments. In this paper, Mathematica ${ }^{\circledR}$ is used to calculate $\Psi$. Alternatively, it is also possible to use the continued fraction on p. 326 in [15], which can in principle be used to calculate $\Psi$ with a desired accuracy using truncation. However, selecting a priori the number of terms necessary to arrive at a desired accuracy is not easy.

The procedure presented here checks the quality of the approximation with respect to its underlying model. It does not give any validation of the true $\chi$ as it depends on many other factors such as variation of the profiles, boundary conditions, approximation $A^{\prime} / A$ and $\phi^{\prime}$, disturbances such as noise, and non-linearities. Noise and non-linearities require an extensive study based on statistics and higher harmonics, which is considered to be beyond the scope of this paper. The problem of varying profiles, approximation $A^{\prime} / A$ and $\phi^{\prime}$, and boundary conditions is studied in [1].

\section{SUMMARY AND DISCUSSION}

In this paper, the problem of determining the diffusion coefficient from measurements during power modulation experiments has been revisited. A large number of new approximations have been introduced to estimate $\chi$ directly from $A^{\prime} / A$ and $\phi^{\prime}$ for different combinations of $\chi, V$, and $\tau_{i n v}$. The approximations are based on infinite domains and are derived on the basis of cylindrical geometry using standard assumptions. These approximations, the approximations from the literature, and those of Part 1 of this paper series have been compared for heat waves traveling outwards (towards the edge). 
The quality of the approximations is presented in several figures. In case only $\chi$ and $\tau_{\text {inv }}$ are considered $(V=0)$, the relative error of the $\chi$ estimate for the region of interest is in general $1 \%$. However, in a small region the errors are larger with a maximum relative error of $20 \%$. These errors are achievable by combining $\chi_{K j 3}$ and $\chi_{A E \Psi}$. In case also $V$ is considered, the new approximations show a significant region in which $\chi$ can be estimated well, but also regions in which no suitable approximation exists. In this case, combining $\chi_{A E \Psi}$ and $\chi_{\phi}$ covers a large region where $\chi$ can be well estimated.

Several figures present the ranges where $\chi$ can be estimated well as function of $\rho, \omega$, and the transport coefficients. However, in practice these are less useful as the true transport coefficients are unknown. Therefore, in this paper a test is presented to check if the chosen approximation reproduces the original $A^{\prime} / A$ and $\phi^{\prime}$. This means that without using knowledge of the actual transport coefficients a proper approximation can be selected. Therefore, it is also necessary to determine the convectivity and damping.

In this paper, the estimation of $\chi$ in a cylindrical geometry using several approximations based on perturbative measurements for heat waves traveling outwards have been discussed. In Part 3, approximations to estimate $\chi$ are derived and discussed for heat waves traveling towards the center (inwards) using either semi-infinite domain assumptions or a symmetry boundary condition [22]. The latter is the natural boundary condition for a cylindrical geometry.

\section{ACKNOWLEDGMENTS}

The first author is currently an International Research Fellow of the Japan Society for the Promotion of Science and hence wishes to express his gratitude to the JSPS for making this research possible. This project has received funding from the European Union's Horizon 2020 research and innovation programme under grant agreement number 633053. The views and opinions expressed herein do not necessarily reflect those of the European Commission. This work is also supported by NWO-RFBR Centre-of-Excellence on Fusion Physics and Technology (Grant nr. 047.018.002). 


\section{Approximations based on continued fractions}

Continued J-fraction of the ratio of Bessel functions of the second kind

The continued $J$-fraction of the ratio between $K_{1}(z) / K_{0}(z)$ is given in (14) and is based on p. 364 in [15]. It is used to calculate three approximations of which two are given in (17) and (21). The approximation considering two terms is given by

$$
\frac{\Theta^{\prime}}{\Theta}=-z-\frac{1}{2 \rho}+\frac{1}{\rho}\left(\frac{1 / 4}{2 z \rho+2}\right)
$$

where $z=\sqrt{\frac{3}{2} \frac{i \omega+\tau_{i n v}}{\chi}}$ and $\Theta^{\prime} / \Theta$ is defined according to $\sqrt{9}$. It can be written in polynomial form, i.e.,

$$
0=8 \rho^{2} z^{2}+\left(8 \rho^{2} \frac{\Theta^{\prime}}{\Theta}+4 \rho\right) z+8 \frac{\Theta^{\prime}}{\Theta} \rho-3
$$

This polynomial is solved in terms of $z$ to calculate $\chi$ (see Table I).

Continued C-fraction of the ratio of Bessel functions of the second kind

The following continued $C$-fraction of the ratio $K_{1}(z) / K_{0}(z)$ is based on p. 363 in [15]

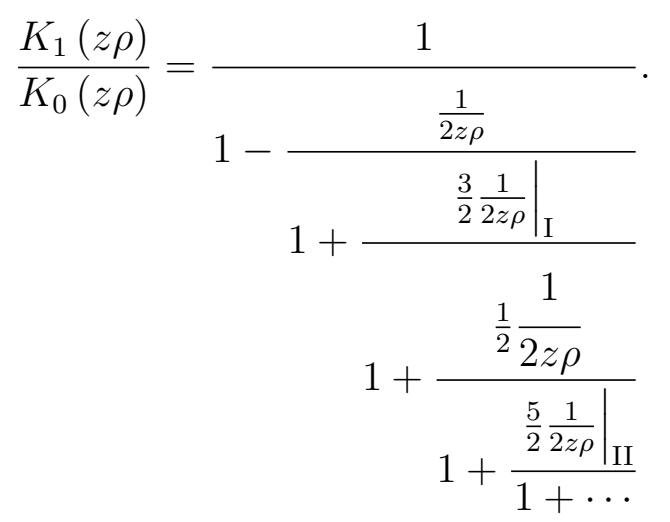

It is used to approximate (11). Here, it is chosen to truncate only at locations I and II to reduce the number of approximations. If 49 is truncated at location I, this results in the following polynomial in terms of $z$

$$
0=4 \rho z^{2}+\left(3+4 \frac{\Theta^{\prime}}{\Theta} \rho\right) z+\frac{\Theta^{\prime}}{\Theta}
$$

which needs to be solved to find $\chi$ and $\tau_{i n v}$. In case 49 is truncated at location II, it gives the following polynomial, which can also be used to find $\chi$ and $\tau_{i n v}$

$$
0=16 \rho^{2} z^{3}+\left(36 \rho+16 \rho^{2} \frac{\Theta^{\prime}}{\Theta}\right) z^{2}+\left(15+28 \rho \frac{\Theta^{\prime}}{\Theta}\right) z+3 \frac{\Theta^{\prime}}{\Theta} .
$$


2. Approximations for $\chi$ only $\left(V=\tau_{i n v}=0\right)$

Consider again (16)

$$
z=-\left(\frac{A^{\prime}}{A}+i \phi^{\prime}+\frac{1}{2 \rho}\right)
$$

Substituting $z$ and expanding

$$
\frac{3}{2} \frac{i \omega}{\chi}=\left(\frac{A^{\prime}}{A}+\frac{1}{2 \rho}\right)^{2}+2 i \phi^{\prime}\left(\frac{A^{\prime}}{A}+\frac{1}{2 \rho}\right)-\left(\phi^{\prime}\right)^{2} .
$$

If the imaginary part is rewritten in terms of $\chi$, it results in

$$
\chi_{s 1}=\frac{3}{4} \frac{\omega}{\left(\phi^{\prime}\right)^{2}} .
$$

This variation was also derived in [18] based on Bessel functions and can be derived in slab-geometry. The real part gives

$$
\chi_{K j 1 A}=\frac{3 \omega}{4\left(\frac{A^{\prime}}{A}+\frac{1}{2 \rho}\right)^{2}} .
$$

The continued fractions with more terms lead to a mix between amplitude and phase, which are complicated to solve. Hence, they are not calculated here.

\section{Asymptotic expansions based on the Bessel function of the second kind}

From [17] the asymptotic expansion for $z \rho \approx 0$ and fixed integer order $\nu \neq 0$ is given by

$$
K_{\nu} \sim \frac{1}{2} \Gamma(\nu)\left(\frac{1}{2} z \rho\right)^{-\nu}
$$

and for order $\nu=0$ is given by

$$
K_{0} \sim-\ln (z \rho)
$$

These can be substituted into (11) to describe $\Theta^{\prime} / \Theta$ around $z \rho \approx 0$

$$
\frac{\Theta^{\prime}}{\Theta}=-z \frac{\left(\frac{1}{2} \Gamma(1)\left(\frac{1}{2} z \rho\right)^{-1}\right)}{(-\ln (z \rho))} .
$$

Simplifying yields

$$
\frac{\Theta^{\prime}}{\Theta}=\frac{1}{\rho \ln (z \rho)}
$$


This can again be expressed in terms of $z$

$$
z=\rho^{-1} \exp \left(\left(\rho \frac{\Theta^{\prime}}{\Theta}\right)^{-1}\right),
$$

which can be solved using the techniques given in Table I

[1] M. van Berkel, H. J. Zwart, N. Tamura, G. M. D. Hogeweij, S. Inagaki, M. R. de Baar, and K. Ida, "Explicit approximations to estimate the perturbative diffusivity in the presence of convectivity and damping (Part 1): Semi-infinite slab approximations," submitted to Physics of Plasmas, vol. -, pp. -, 2014.

[2] N. J. Lopes Cardozo, "Perturbative transport studies in fusion plasmas," Plasma Phys. Control. Fusion, vol. 37, p. 799, 1995.

[3] S. P. Eury, E. Harauchamps, X. Zou, and G. Giruzzi, "Exact solutions of the diffusionconvection equation in cylindrical geometry," Physics of Plasmas, vol. 12, p. 102511, 2005.

[4] A. Jacchia, P. Mantica, F. De Luca, and G. Gorini, "Determination of diffusive and nondiffusive transport in modulation experiments in plasmas," Physics of Fluids B, vol. 3, no. 11, pp. 30333040, 1991.

[5] E. von Kamke, Differentialgleichungen: Loesungsmethoden und Loesungen. Geest \& Portig, Leipzig, 1959.

[6] A. D. Polyanin and V. F. Zaitsev, Handbook of Exact Solutions for Ordinary Differential Equations. Chapman \& Hall/CRC, London, 2003, vol. 2.

[7] H. Bateman and A. Erdelyi, Higher transcendental functions, A. Erdelyi, Ed. Mcgraw-Hill, New York, 1953.

[8] L. Slater, Confluent hypergeometric functions. Cambridge University Press, Cambridge, 1960.

[9] Y. L. Luke, The special functions and their approximations, Volume 1, Stegun, Ed. Elsevier, 1969.

[10] H. S. Carslaw and J. C. Jaeger, Conduction of heat in solids. Clarendon Press, Oxford, 1959, vol. 1.

[11] R. Pintelon and J. Schoukens, System Identification: A Frequency Domain Approach. WileyIEEE Press, Hoboken (NJ), 2012.

[12] L. Ljung, System identification. Wiley Online Library, 1999. 
[13] R. F. Curtain and K. Morris, "Transfer functions of distributed parameter systems: A tutorial," Automatica, vol. 45, no. 5, pp. 1101-1116, 2009.

[14] W. B. Jones and W. T. C. Fractions, Analytic Theory and Applications, ser. Encyclopedia of Mathematics and its Applications. Addison-Wesley Publishing Company, London, 1980, vol. 11 .

[15] A. Cuyt, V. B. Petersen, B. Verdonk, H. Waadeland, and W. B. Jones, Handbook of continued fractions for special functions. Springer Netherlands, 2008.

[16] A. Erdélyi, Asymptotic expansions. Courier Dover Publications, NY, 1956.

[17] M. Abramowitz and I. Stegun, Handbook of mathematical functions: with formulas, graphs, and mathematical tables. Dover, New York, 1976.

[18] E. Fredrickson, J. Callen, K. McGuire, J. Bell, R. Colchin, P. Efthimion et al., "Heat pulse propagation studies in TFTR," Nuclear Fusion, vol. 26, no. 7, p. 849, 1986.

[19] M. Soler and J. Callen, "On measuring the electron heat diffusion coefficient in a tokamak from sawtooth oscillation observations," Nuclear Fusion, vol. 19, no. 6, p. 703, 1979.

[20] M. van Berkel, H. J. Zwart, G. M. D. Hogeweij, G. Vandersteen, H. van den Brand, M. R. de Baar, and the ASDEX Upgrade Team, "Estimation of the thermal diffusion coefficient in fusion plasmas taking frequency measurement uncertainties into account," (accepted for publication in Plasma Phys. Control. Fusion), vol. (-), pp. (-), 2014.

[21] N. L. Cardozo, B. Tubbing, F. Tibone, and A. Taroni, "Heat pulse propagation: Diffusive models checked against full transport calculations," Nuclear Fusion, vol. 28, no. 7, p. 1173, 1988.

[22] M. van Berkel, N. Tamura, H. J. Zwart, G. M. D. Hogeweij, S. Inagaki, M. R. de Baar, and K. Ida, "Explicit approximations to estimate the perturbative diffusivity in the presence of convectivity and damping (Part 3): Cylindrical approximations for heat waves traveling inwards," submitted to Physics of Plasmas, vol. -, pp. -, 2014. 